\title{
Real-time Zeta Potential Analysis of Microchannel Surfaces during Aminosilane Deposition and Exposure Using Current Monitoring
}

\author{
Austin S. Abrams ${ }^{\dagger}$, Alexander Eden ${ }^{\ddagger}$, Lingyun Zhou ${ }^{\ddagger}$, Bing Wang $^{\ddagger}$, David E. Huber ${ }^{\ddagger}$, and Sumita Pennathur ${ }^{*}$, \\ ${ }^{\dagger}$ Department of Chemistry and Biochemistry, University of California, Santa Barbara, Santa Barbara, California 93106, \\ United States \\ ${ }^{\ddagger}$ Department of Mechanical Engineering, University of California, Santa Barbara, Santa Barbara, California 93106, United \\ States
}

${ }^{*}$ Corresponding Author:

Sumita Pennathur, Professor of Engineering

Engineering Science Building, Room 3231C

University of California, Santa Barbara,

Santa Barbara, CA 93106-5070

Email: sumita@engineering.ucsb.edu

Phone: +1 (805) 893-5510

Other Authors:

Austin S.Abrams: austin.abrams@chem.ucsb.edu

Alexander Eden: a_eden@ucsb.edu

Lingyun Zhou: zhouly@ucsb.edu

Bing Wang: bwang@chem.ucsb.edu

David E.Huber: dhuber@ucsb.edu

\section{Table of Contents}

1. System Design $\quad$ S2

2. Temporal Resolution of Buffer Exchanges S2-S3

3. Zeta Potential vs $\mathrm{pH}$ of Bare and APTMS-Coated Capillaries S3-S5

4. Zeta Potential vs Time of APS Coating Formations and Exposures S5-S7

$\begin{array}{ll}\text { References } & \text { S7 }\end{array}$ 


\section{System Design}

The study of surface reactions imposes more stringent requirements than are needed for single-shot measurements in equilibrium conditions. Fluid conditions within the reservoirs must be relatively stable over the length of the reaction (e.g., hours to days), and native surfaces are required for each instance. We use high-volume reservoirs (relative to the capillary) that were capped to maintain the solution composition and minimize transport-induced mixing over long operating periods.

We chose a channel diameter of $1.2 \mu \mathrm{m}$ to minimize Joule heating ${ }^{1}$ and pressure-driven flow resulting from unintentional differences in fluid levels between reservoirs. We employed an $8 \mathrm{~mm}$ channel length to increase measurement frequency while reducing the relative influence of diffusion compared to advective transport under the applied electric field and concentration gradient. Our channel design has the advantages that it is constructed from commercially available capillary tubing and requires minimal fabrication; this design allows channels to be prepared in bulk and at low cost.

\section{Temporal Resolution of Buffer Exchanges}

To assess the temporal response of automated zeta potential analysis (AZA) compared to the known reaction time of around $15 \mathrm{~s}$ for $\mathrm{pH}$-based surface protonation of silica gels, ${ }^{2}$ we performed additional buffer and $\mathrm{pH}$ exchange experiments (Figure S1). Unlike conventional analysis of a substrate immersed in a large beaker of solution, AZA requires solution to be displaced through the capillary before surface reactions can occur. We found that the time to reach stable zeta potential $(\zeta)$ values after a $\mathrm{pH}$ or buffer exchange was related to the time to displace solution from the capillary, as the traversal time is inversely proportional to the applied electric field strength and EOF velocity. This stabilization time was 38-72 s for buffer exchange experiments where $\mathrm{E}_{0}=30 \mathrm{kV} / \mathrm{m}$, while at $60 \mathrm{kV} / \mathrm{m}$ this time was reduced to 23-27 s (Table S1). Even with a delay time of $72 \mathrm{~s}$ at $30 \mathrm{kV} / \mathrm{m}, \mathrm{AZA}$ has sufficient temporal resolution to extract the kinetics of many reactions which occur at slower rates than surface protonation.

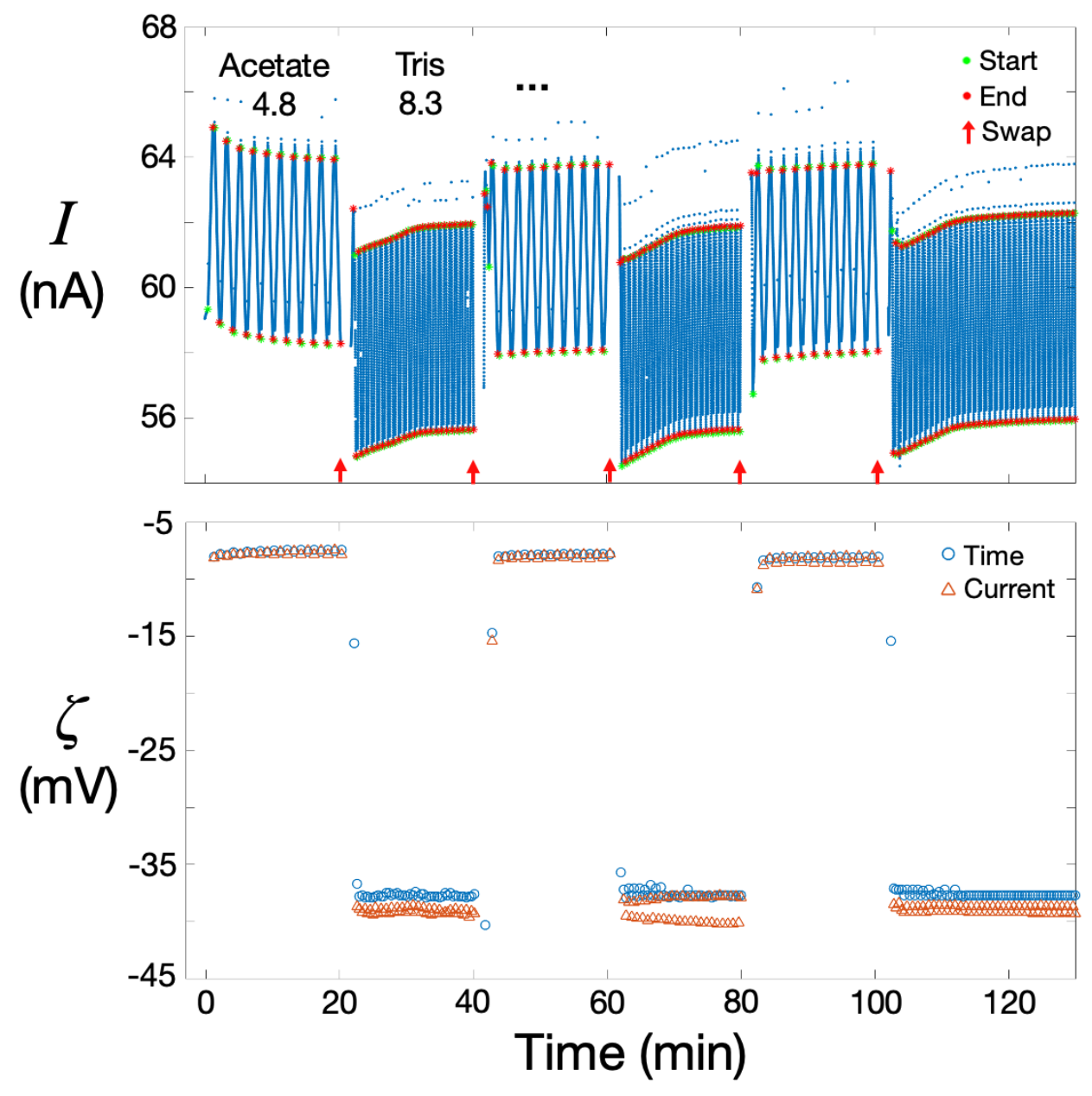

Figure S1. Current monitoring cycles and calculated zeta potential response as an unconditioned capillary was exposed to $\mathrm{pH} 4.8$ acetate or $\mathrm{pH} 8.3$ Tris buffered saline solutions. Measured conductivities were 1.69 and 1.63 
$\mathrm{S} / \mathrm{m}$, respectively. $1 \times$ solutions consisted of $20 \mathrm{mM}$ buffer and $150 \mathrm{mM} \mathrm{NaCl}$. Zeta potentials were calculated using traversal time (eq 3 ) or the slope method by Sze et al. ${ }^{3}$

Table S1. Time for Zeta Potential Readings to Stabilize after Various In Situ Buffer Exchanges (Fig S1).

\begin{tabular}{|c|c|c|c|}
\hline $\begin{array}{l}\text { Electric Field Strength } \\
\qquad(\mathrm{kV} / \mathrm{m})\end{array}$ & $\begin{array}{l}\text { Buffers in Exchange } \\
\text { (A to B) }\end{array}$ & $\begin{array}{c}\text { Observed Stabilization } \\
\text { Time }(\mathrm{s})^{1}\end{array}$ & $\begin{array}{c}\text { Standard } \\
\text { Deviation }(s)\end{array}$ \\
\hline 60 & MES to Tris & 23 & \pm 3 \\
\hline 60 & Tris to MES & 27 & \pm 1 \\
\hline 30 & MES to Tris & 38 & \pm 1 \\
\hline 30 & Tris to MES & 72 & \pm 4 \\
\hline 30 & Acetate to Phosphate & 38 & \pm 2 \\
\hline 30 & Phosphate to Tris & 35 & \pm 2 \\
\hline 30 & Tris to Acetate & 86 & \pm 2 \\
\hline
\end{tabular}

${ }^{1}$ Average time until the deviation was less than $2 \mathrm{mV}$ consecutive zeta potential readings.

\section{Zeta Potential vs pH of Bare And APTMS-Coated Capillaries}

Table S2. Time-Averaged Equilibrium Zeta Potentials of a Bare Capillary in Varying pH Solutions (Fig 2c).

\begin{tabular}{|c|c|c|c|c|c|c|c|}
\hline $\mathrm{pH}$ & $\stackrel{\mathcal{\zeta}}{(\mathrm{mV})^{1}}$ & $\begin{array}{l}\text { Confidence } \\
\text { Interval, 95\% } \\
( \pm \mathrm{mV})\end{array}$ & $\begin{array}{c}\text { Sample } \\
\text { Size }^{2}\end{array}$ & Buffer & $\begin{array}{c}\text { Electric Field } \\
\text { Strength } \\
(\mathrm{kV} / \mathrm{m})\end{array}$ & $\begin{array}{l}\text { Conductivity } \\
(\mathrm{S} / \mathrm{m})\end{array}$ & Day $^{3}$ \\
\hline 2.19 & 0.6 & 0.1 & 6 & Phosphate & 123 & 1.63 & 1 \\
\hline 7.11 & -26.5 & 0.1 & 37 & Phosphate & 30 & 1.63 & 1 \\
\hline 3.11 & 0.8 & 0.2 & 6 & Citrate & 120 & 1.63 & 1 \\
\hline 8.38 & -34.9 & 0.1 & 19 & Tris & 30 & 1.63 & 1 \\
\hline 4.77 & -3.7 & 0.1 & 5 & Acetate & 30 & 1.63 & 1 \\
\hline 9.55 & -35.4 & 0.1 & 20 & CHES & 30 & 1.63 & 1 \\
\hline 6.16 & -16.0 & 0.1 & 10 & MES & 30 & 1.63 & 1 \\
\hline 10.37 & -38.2 & 0.1 & 9 & CAPS & 30 & 1.63 & 1 \\
\hline 4.84 & -2.9 & 0.1 & 4 & Citrate & 30 & 1.63 & 2 \\
\hline 5.50 & -5.9 & 0.1 & 10 & Citrate & 30 & 1.63 & 2 \\
\hline 3.99 & -1.1 & 0.2 & 9 & Citrate & 90 & 1.63 & 2 \\
\hline 2.14 & 0.8 & 0.1 & 7 & Phosphate & 30 & 1.63 & 1 \\
\hline 3.07 & 0.5 & 0.1 & 3 & Citrate & 30 & 1.63 & 1 \\
\hline 3.85 & -0.8 & 0.1 & 4 & Citrate & 30 & 1.63 & 1 \\
\hline
\end{tabular}




\begin{tabular}{|c|c|c|c|c|c|c|c|}
\hline 4.72 & -2.8 & 0.1 & 8 & Citrate & 30 & 1.63 & 1 \\
\hline 5.34 & -4.9 & 0.1 & 257 & Citrate & 30 & 1.63 & 1 \\
\hline 6.12 & -12.4 & 0.4 & 24 & MES & 30 & 1.63 & 2 \\
\hline 7.05 & -24.4 & 0.5 & 746 & Phosphate & 30 & 1.63 & 2 \\
\hline 8.36 & -31.4 & 0.3 & 284 & Tris & 30 & 1.63 & 3 \\
\hline 6.67 & -16.6 & 0.1 & 17 & Bis-Tris & 30 & 1.63 & 3 \\
\hline 7.59 & -26.5 & 0.1 & 8 & HEPES & 30 & 1.63 & 3 \\
\hline 9.47 & -35.0 & 0.7 & 2189 & CHES & 30 & 1.63 & 3 \\
\hline 10.25 & -33.3 & 0.6 & 21 & CAPS & 30 & 1.63 & 3 \\
\hline 12.62 & -35.6 & 0.7 & 11 & Phosphate & 30 & 1.63 & 3 \\
\hline 5.00 & -2.7 & 0.1 & 8 & Acetate & 20.9 & 1.63 & 1 \\
\hline 5.34 & -3.4 & 0.1 & 7 & Acetate & 20.9 & 1.64 & 1 \\
\hline 5.85 & -5.8 & 0.1 & 6 & Acetate & 20.9 & 1.66 & 1 \\
\hline 5.77 & -6.3 & 0.1 & 5 & Phosphate & 20.9 & 1.61 & 2 \\
\hline 6.12 & -8.6 & 0.5 & 4 & Phosphate & 20.9 & 1.62 & 2 \\
\hline 6.45 & -13.0 & 0.2 & 17 & Phosphate & 20.9 & 1.64 & 2 \\
\hline 6.75 & -15.8 & 0.1 & 6 & Phosphate & 20.9 & 1.65 & 2 \\
\hline 7.07 & -18.5 & 0.5 & 5 & Phosphate & 20.9 & 1.66 & 2 \\
\hline 7.28 & -20.2 & 0.7 & 5 & Phosphate & 20.9 & 1.67 & 2 \\
\hline 7.61 & -23.0 & 1.4 & 3 & Phosphate & 20.9 & 1.68 & 2 \\
\hline 7.93 & -24.9 & 0.5 & 6 & Phosphate & 20.9 & 1.70 & 2 \\
\hline 8.12 & -26.0 & 0.2 & 2 & Borate & 20.9 & 1.55 & 2 \\
\hline 8.41 & -28.3 & 0.3 & 4 & Borate & 20.9 & 1.57 & 2 \\
\hline 8.73 & -33.7 & 0.1 & 4 & Borate & 20.9 & 1.60 & 4 \\
\hline 8.98 & -35.2 & 0.1 & 6 & Borate & 20.9 & 1.63 & 5 \\
\hline 9.27 & -37.3 & 0.1 & 9 & Borate & 20.9 & 1.65 & 5 \\
\hline 9.54 & -39.0 & 0.2 & 7 & Borate & 20.9 & 1.67 & 5 \\
\hline 9.91 & -39.3 & 0.1 & 10 & Borate & 20.9 & 1.70 & 5 \\
\hline 10.17 & -38.8 & 0.4 & 3 & Borate & 20.9 & 1.72 & 5 \\
\hline 10.47 & -38.3 & 0.5 & 3 & Borate & 20.9 & 1.75 & 6 \\
\hline 12.00 & -34.9 & 0.2 & 18 & Phosphate & 20.9 & 1.95 & 7 \\
\hline
\end{tabular}

${ }^{1}$ Time-averaged zeta potential after reaching equilibrium (less than $2 \mathrm{mV}$ deviation between readings).

${ }^{2}$ Number of zeta potentials used to calculate time-averaged value.

${ }^{3}$ Individual titration series from Figure $2 \mathrm{c}(1: \mathbf{\square}, 2: \bullet, 3: \bullet)$ are listed chronologically and separated by horizontal lines. Capillary size $(\mathrm{d}$ $\times \mathrm{L}): 1.2 \mu \mathrm{m} \times 8.0 \mathrm{~mm}(\boldsymbol{\bullet} / \bullet), 1.1 \mu \mathrm{m} \times 23.9 \mathrm{~mm}(\bullet)$. Buffer concentration: $20 \mathrm{mM}(\boldsymbol{\bullet} / \bullet), 10 \mathrm{mM}(\bullet)$. 
Table S3. Solution measurements of APTMS capillary exposures to varying pH (Fig 3b).

\begin{tabular}{clccccc}
$\mathrm{pH}$ & Buffer & $\xi_{\text {bare }}$ & $\xi_{0 \mathrm{~h}}$ & $\xi_{36 \mathrm{~h}}$ & $\sigma_{0.9 \times}$ & $\sigma_{1 \times}$ \\
2.10 & Phosphate & 4.4 & 58.4 & 57.1 & 1.44 & 1.60 \\
4.86 & Acetate & -5.3 & 44.3 & 35.6 & 1.55 & 1.69 \\
6.04 & Phosphate & -12.4 & 28.7 & 13.6 & 1.43 & 1.58 \\
7.12 & Phosphate & -24.4 & 15.7 & -9.0 & 1.43 & 1.58 \\
8.33 & Tris & -30.4 & 14.7 & -20.5 & 1.49 & 1.63 \\
9.55 & CHES & -35.5 & -7.9 & -34.4 & 1.44 & 1.59 \\
10.53 & CAPS & -37.5 & -18.1 & -35.6 & 1.46 & 1.60 \\
\hline
\end{tabular}

\section{Zeta Potential vs Time of APS Coating Formations and Exposures}

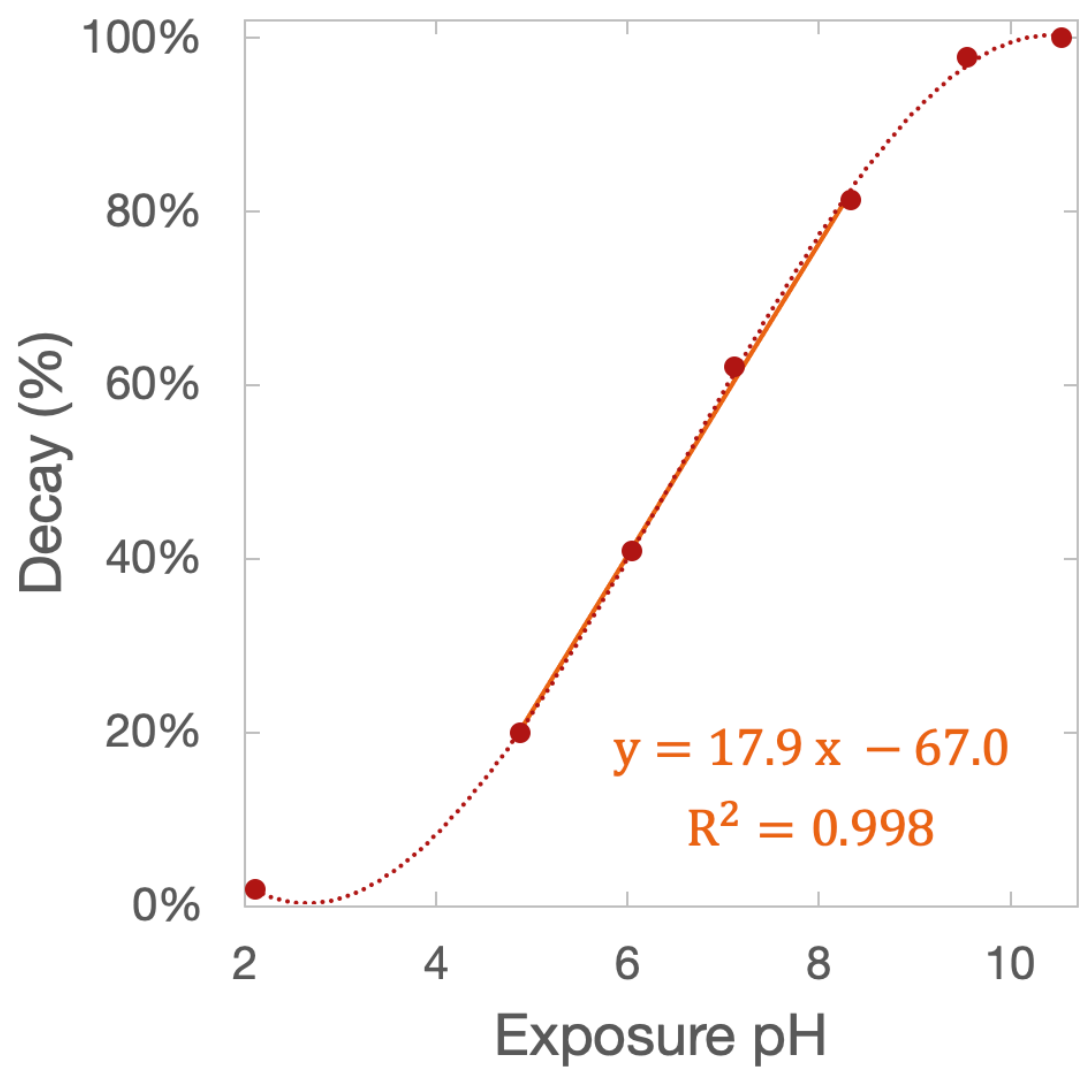

Figure S2. Normalized zeta potential decay after $36 \mathrm{~h}$ exposure of APTMS-coated capillaries to varying $\mathrm{pH}$ solutions (Table S3). A linear fit shows the decay rate from $\mathrm{pH} 4.9$ to 8.3. 

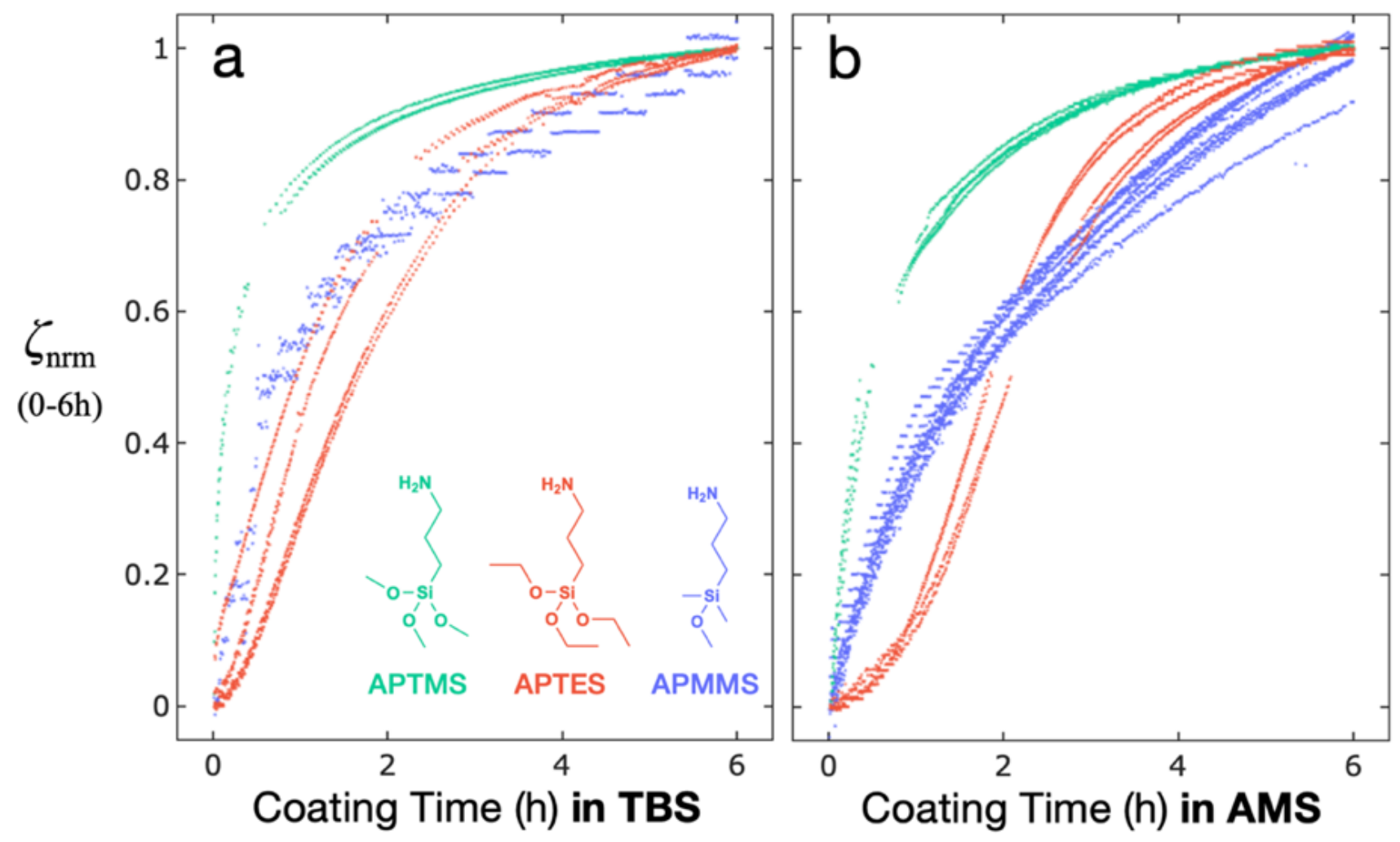

Figure S3. Normalized zeta potential response of $1 \mathrm{mM}$ coating formations in (a) TBS or (b) AMS, calculated by dividing the change in zeta potential from 0 to time, $t$, by the change from 0 to $6 \mathrm{~h}$.

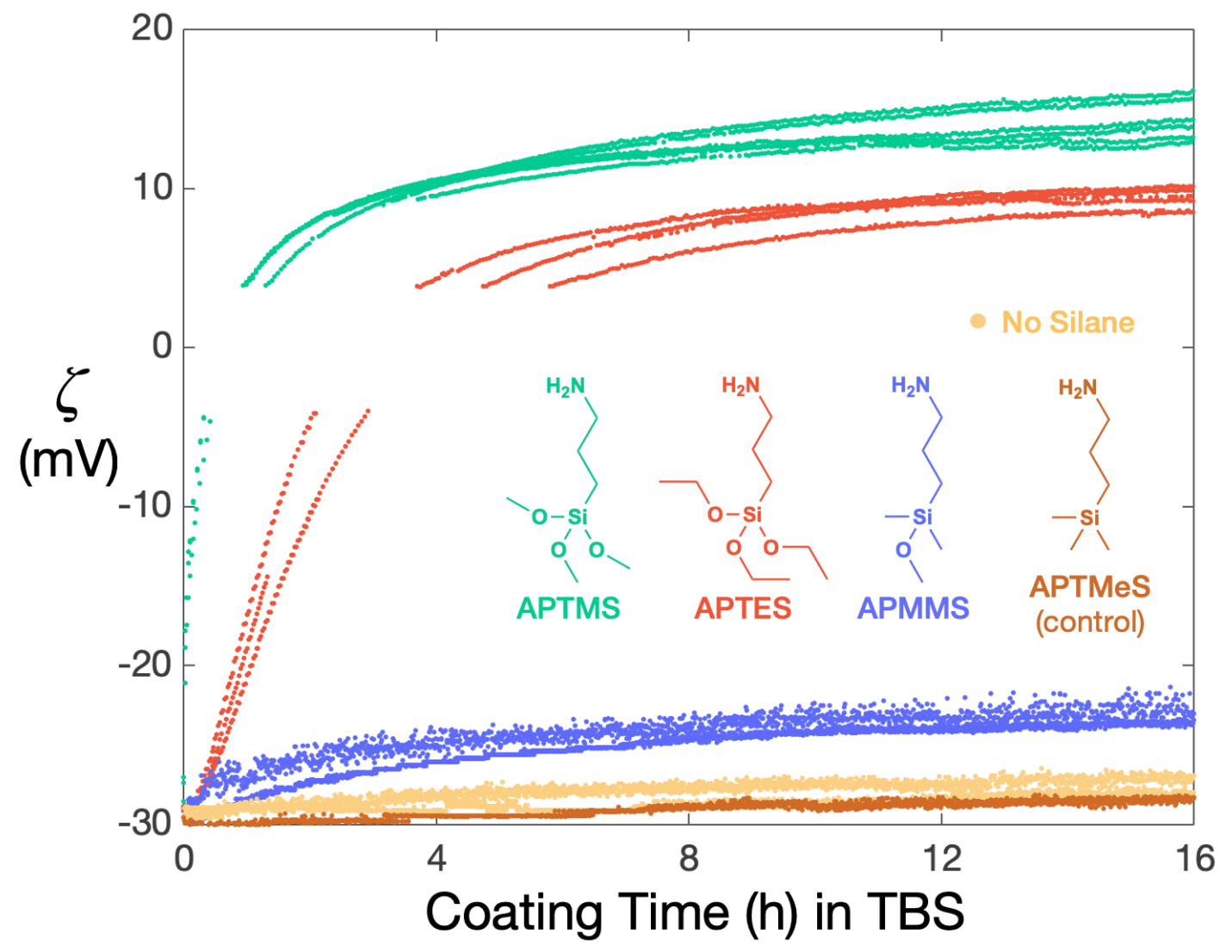


Figure S4. Zeta potential response over $16 \mathrm{~h}$ coating formations, with 3-aminopropyltrimethylsilane and silanefree TBS controls. At $0 \mathrm{~h}$, APS was added to $0.9 \times / 1 \times$ TBS reservoirs at $1 \mathrm{mM}$ concentration.

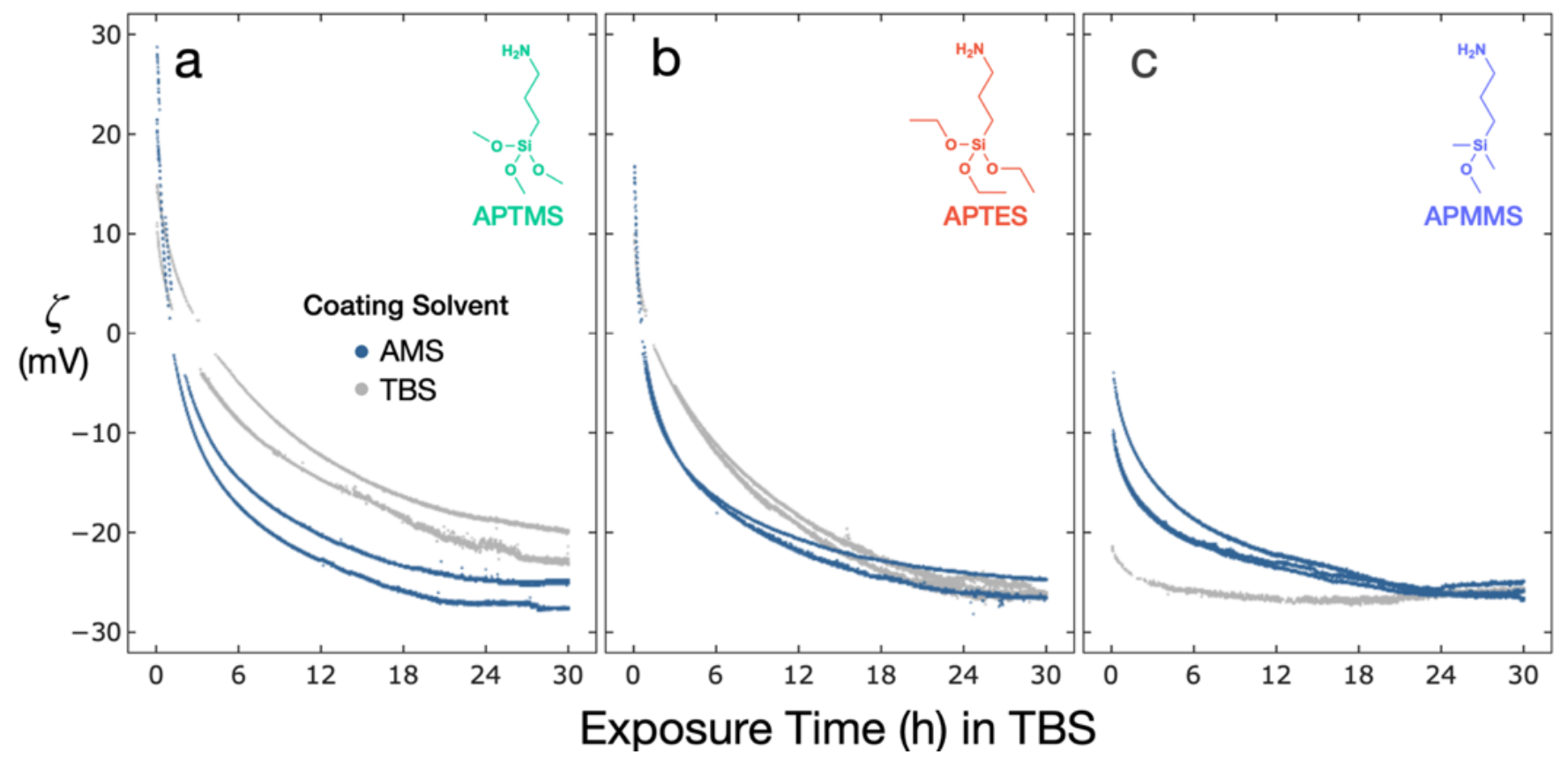

Figure S5. Raw zeta potential response of APS coatings exposed to silane-free, $\mathrm{pH}$ 8.3 TBS. Coatings were formed over $6 \mathrm{hm}$ with $1 \mathrm{mM}$ respective monomer in TBS or AMS reservoirs.

\section{References}

(1) Tang, G. Y.; Yang, C.; Chai, J. C.; Gong, H. Q. Joule Heating Effect on Electroosmotic Flow and Mass Species Transport in a Microcapillary. International Journal of Heat and Mass Transfer 2004, 47 (2), 215-227.

(2) Walcarius, A.; Etienne, M.; Bessière, J. Rate of Access to the Binding Sites in Organically Modified Silicates. 1. Amorphous Silica Gels Grafted with Amine or Thiol Groups. Chem. Mater. 2002, 14 (6), 2757-2766.

(3) Sze, A.; Erickson, D.; Ren, L.; Li, D. Zeta-Potential Measurement Using the Smoluchowski Equation and the Slope of the Current-Time Relationship in Electroosmotic Flow. J. Colloid Interface Sci. 2003, 261 (2), 402-410. 